\title{
Calculation of Surrounding Rock Pressure of Undercut Subway Station Based on Multi-Factor Affecting Pressure Arch Theory
}

\author{
Bin Shang1, Xiaoguang Jin ${ }^{2 *}$, Guiyong Ao ${ }^{1}$, Qiao Chen ${ }^{2}$, Qiong Qiu ${ }^{1}$ \\ ${ }^{1}$ China Construction Tunnel Construction Co. Ltd., Chongqing, China \\ ${ }^{2}$ School of Civil Engineering, Chongqing University, Chongqing, China \\ Email: `jxgcqu@163.com
}

How to cite this paper: Shang, B., Jin, X.G., Ao, G.Y., Chen, Q. and Qiu, Q. (2020) Calculation of Surrounding Rock Pressure of Undercut Subway Station Based on Multi-Factor Affecting Pressure Arch Theory. Engineering, 12, 59-70.

https://doi.org/10.4236/eng.2020.122006

Received: December 20, 2019

Accepted: January 19, 2020

Published: January 22, 2020

Copyright $\odot 2020$ by author(s) and Scientific Research Publishing Inc. This work is licensed under the Creative Commons Attribution International License (CC BY 4.0).

http://creativecommons.org/licenses/by/4.0/

\begin{abstract}
The formation mechanism of surrounding rock pressure in large-section tunnels of underground excavation is still unclear. The traditional methods for calculating surrounding rock pressure have certain limitations. Based on the theory of pressure arch and parameter sensitivity analysis, the internal and external boundaries of the pressure arch are solved by numerical simulation. The loose pressure is obtained according to the distance from the inner boundary to the inner wall of the tunnel. The pressure arch deformation is analyzed based on the Winkler model. The surrounding rock pressure is calculated, and the surrounding rock pressure based on the pressure arch theory is calculated. The calculation method of surrounding rock pressure based on the pressure arch theory large section tunnel is proposed. The orthogonal experimental design method was used to select four representative factors: tunnel depth $\mathrm{H}$, span $\mathrm{B}$, internal friction angle $\varphi$ and rock weight $\gamma$. The calculation results of each group of experiments were compiled and multivariate linear regression was used. The method is statistically analyzed, and finally, the calculation formula of the surrounding rock pressure of the large-section tunnel affected by multiple factors is obtained and applied to the calculation of the surrounding rock pressure of the metro station.
\end{abstract}

\section{Keywords}

Pressure Arch Theory, Sensitivity Analysis, Surrounding Rock Pressure, Orthogonal Test, Numerical Simulation

\section{Introduction}

The commonly used theory of surrounding rock pressure includes the theory of 
whole soil column, Platts theory, Terzaghi theory, Xie Jiaxiao theory, Bill Bowman theory, etc. [1] [2] [3]. In platt's theory, the upper part of the tunnel arch needs to form a stable natural balance arch, the range of rock mass acting on the supporting structure is obtained through its position and the height of the arch body, and the rock mass firmness coefficient $f$ is introduced. $f$ is not a characteristic parameter of rock mass, and its value needs to be determined by the value of normal stress $\sigma$, which is difficult to be obtained through experiments. In practical application, $f$ is usually determined by empirical formula. Based on the principle of stress transfer and soil pressure, Terzaghi theory calculates the overall stress situation of the rock mass in the upper sliding zone of the tunnel by analyzing the stress balance of the differential soil stripes, and calculates the lateral pressure of the soil column during the slide by combining the lateral pressure coefficient and Mohr-Coulomb law. On the basis of a large number of experiments, it is recommended that the lateral pressure coefficient should be 1.0, but in practical engineering, it is inevitable that it does not conform to the value, which leads to some errors in the application of the theory. Xie Jiaxiao theory in the theoretical derivation based on the principle of limit equilibrium of soil, the force balance of the rock pillar and triangular rock mass on both sides of the calculation model is solved respectively, the friction resistance of the rock mass on the upper part of the tunnel is calculated by mathematical and mechanical methods, the parameter $\lambda, \beta, \theta$ and so on were introduced in the process of theoretical derivation. $\theta$ angle and calculated friction Angle can only be evaluated according to a certain range given by the code, which are difficult to obtain in engineering practice. This makes Xie Jiaxiao theory have some limitations when applied to the surrounding rock pressure of tunnel engineering solution. Similar to the Terzaghi theory, the lateral friction resistance on the element body in Bill Bowman theory is determined by Mohr-Coulomb law, and then the differential friction resistance is integrated to obtain the overall friction resistance. The vertical surrounding rock pressure on the upper part of the tunnel can be obtained by means of stress balance analysis. However, in practical application, when the surrounding rock pressure is calculated by Terzaghi theory and Bill Bowman theory, negative values may occur due to the large cohesion of the rock mass, which is inconsistent with the actual situation.

Pressure arch is a kind of real arch structure, which is difficult to be observed in actual engineering. The pressure arch effect is derived from the stress field of underground engineering rock mass. During tunnel excavation and supporting construction, the rock mass around the tunnel is disturbed due to excavation, and an arch structure resisting such disturbing deformation will be produced within a certain range of the surrounding rock.

The load of the surrounding rock transfers through the arch structure which forms a self-adjusting stable state between the load generated by the tunnel excavation and the rock mass itself. When a pressure arch is formed in the surrounding rock of the tunnel, the principal stress on the tunnel vault path will be deflected in direction and its stress value will also change. The load will be 
transferred to both sides of the tunnel and eventually reach the stable rock mass at the arch springing. Due to the similar action of tangential wedge in the surrounding rock, the load will gradually become stable.

After the tunnel excavation, stress mutation will occur in the surrounding rock due to the disturbance applied to the in-situ stress field, resulting in stress concentration in the rock mass around the tunnel contour. When the mutation stress value exceeds the ultimate bearing capacity of rock mass, the surrounding rock will be destroyed, and the rock mass at the failure point enters a plastic state, and gradually formed the plastic zone of surrounding rock as the deepening of failure. In this interaction, the ultimate bearing capacity of the rock mass is weakened and a stress reduction zone will be formed in a certain area around the tunnel. Surrounding rock stress reduced zone will continue to transfer and expand further with the high-stress area to the depths of the surrounding rock, the stress state of surrounding rock changes from bidirectional stress state to tridirectional stress state. The ultimate bearing capacity of the surrounding rock will gradually increase with the increase of the absolute value of the third principal stress. And after reaching the critical position, its bearing capacity will exceed the stress value of the position, so that the plastic zone of the surrounding rock stops developing and gradually changes to the elastic bearing state.

The range of the stress reduction zone is the range of the loose zone, the loose zone and the surrounding rock in the elastic state are the range of the pressure arch, while the rock mass above the pressure arch body is in the state of the original rock stress.

Ziping Huang et al. [4] made a qualitative analysis of the pressure arch through discrete element (UDEC), but could not determine its precise range. Liang Xiaodan et al. [5] determined the boundary range of the pressure arch based on the Flac 2D software and the stress analysis method, and illustrated it with a single hole with a buried depth of $800 \mathrm{~m}$. Yu bo, Wang Hujia et al. [6] studied the characteristics of pressure arch with ANSYS finite element analysis software, and analyzed the influence of some factors on the formation of pressure arch. Wang Yunfei et al. [7] combined with Flac3D finite difference software to analyze the influence of different excavation methods on pressure arch formation. Li Kui [8] used finite element software (ABAQUS) to study the mechanism of arch formation of horizontal layered tunnel, recommended the determination method of internal and external boundary of pressure arch, and discussed the influence factors of arch formation. C.J. Lee [9] explored the pressure arch range of a single chamber of continuous media through centrifuge test and Flac2D simulation. Jianhong $\mathrm{Wu}$ et al. [10] conducted mechanical behavior simulation of cutters rock mass with DDA numerical method, and qualitatively analyzed the arch shape and development process of surrounding rock pressure with the change of element stress direction as the judgment index. In addition, B. Shen [11], C. Callari [12], R.K. Bhasin et al. [13] and J.B. Martino et al. [14] studied the pressure arch from different angles by using finite element or discrete element. 


\section{Calculation of Surrounding Rock Pressure Based on Pressure Arch Theory}

According to the pressure arch theory, when the tunnel is excavated, the surrounding rock loose zone, the pressure arch body and the original rock stress zone are formed from the inside to outside. The tangential stress in the surrounding rock firstly decreases below the stress value of the original rock, and the stress relaxation occurs in the soil where the stress of this part is less than the stress of the original rock, which can be regarded as the loose pressure caused by the failure of the rock mass and the excavation of the tunnel. This stress force forms part of the surrounding rock pressure. The ultimate bearing capacity of the surrounding rock increases with the depth of the path and exceeds the stress value when it reaches the critical position. Because the stress of rock mass inside the pressure arch is higher than the stress of original rock state before excavation, it will not produce loose pressure. However, due to the action of its own gravity and the pressure of the upper rock mass, this part of rock mass produces certain deformation, and the deformation pressure is transferred to the support structure, forming part of the surrounding rock pressure. Therefore, the surrounding rock pressure is composed of the loose pressure in the loose zone and the pressure caused by the compression deformation of the pressure arch. According to the above discussion on pressure arch, it can be known that the surrounding rock pressure after tunnel excavation consists of two parts:

1) Loose pressure $p_{1}$ in loose zone:

$$
p_{1}=\gamma H_{1}
$$

$H_{1}$ is the distance between the pressure arch inner boundary and the excavation inner wall;

$\gamma$ is the weight of rock mass.

2) Pressure $p_{2}$ generated by compression and deformation of pressure arch:

$$
p_{2}=k s
$$

$s$ is the compression deformation of the pressure arch.

$k$ is the stiffness of the pressure arch.

According to the above theoretical analysis and Equation (1) and Equation (2), it can be deduced that the surrounding rock pressure $\mathrm{p}$ under the pressure arch theory is

$$
p=p_{1}+p_{2}=\gamma H_{1}+k s
$$

According to the judgment criterion of the internal boundary of the pressure arch [4] [5], the thickness of the loose rock mass between the internal boundary of the pressure arch and the inner wall of the tunnel can be obtained and $p_{1}$ can be obtained, so the calculation of surrounding rock pressure also includes the pressure $p_{2}$ generated by the compression deformation of the pressure arch body.

For the calculation of $p_{2}$, based on the Winkler model theory, the surrounding rock above the tunnel vault is simplified and assumed to be a continuous 


$$
k=\left\{\begin{array}{l}
\frac{E}{H} \\
\frac{E}{\left(1-\mu^{2}\right) H} \\
\frac{(1-\mu) E}{(1+\mu)(1-2 \mu) H}
\end{array}\right.
$$

(When both sides of the compressed layer are free to deform)

(When only one side of the compressed layer is free to deform)

(When free deformation is not allowed on either side of the compressed layer)

According to Equation (4), the stiffness $k$ of the pressure arch is:

$$
k=\frac{E}{H_{2}}
$$

$E$ is the elastic modulus of rock mass, $\mathrm{H}_{2}$ is the thickness of pressure arch.

The compression displacement value $s$ of the pressure arch can be obtained by using MIDAS/GTS NX finite element numerical analysis software. Combined with the numerical simulation results and the Judgment criterion of pressure arch boundary, the specific range of the pressure arch can be obtained, the path from the vault to the surface can be defined, and the displacement value of the rock mass in the elastic state can be taken as the compression value $s$. According to the elastic-plastic principle, the displacement value corresponding to the maximum tangential stress of the rock mass is the displacement value $s_{1}$ of the elastomer. $s_{1}$ also includes the overall subsidence displacement of the pressure arch due to excavation, so it cannot be taken as the true displacement of the arch, and the subsidence value of arch body $s_{2}$ should be subtracted.

The displacement value of the middle position of the pressure arch was selected as the overall subsidence value $s_{2}$ of the arch. At the same time, the displacement value of the point also includes the deformation value of the rock mass at the point, that is, the product of the strain value and unit length. To sum up, the deformation $s$ of the pressure arch is: 


$$
s=s_{1}-s_{2}
$$

In Equation (6), $s_{1}$ is the displacement value of the maximum point of tangential stress on the vault path after tunnel excavation; $s_{2}$ is the overall displacement of the pressure arch.

\section{Calculation of Pressure Arch Theory Surrounding Rock Pressure Based on Multi-Factor Influence}

The determination of surrounding rock pressure in engineering practice is related to many factors. For the calculation of surrounding rock pressure under the influence of multiple physical parameters of tunnel surrounding rock, the orthogonal experimental design can be used to carry out the research, and the influence degree of each physical parameter on the calculated value can be analyzed, and the calculation method of surrounding rock pressure based on the pressure arch theory can be simplified into a practical calculation formula.

\subsection{Parameter Sensitivity Analysis}

Parametric sensitivity analysis is a method often used to analyze system stability: Suppose that a characteristic $P$ of the system is mainly related to $\mathrm{n}$ factors, and the relation between this characteristic $P$ and $\alpha$ is a function, and the relation can be expressed as $P=f\left\{\alpha_{1}, \alpha_{2}, \cdots, \alpha_{n}\right\}$. If all the factors in the system are in a certain benchmark state $\alpha^{*}=\left\{\alpha_{1}^{*}, \alpha_{2}^{*}, \cdots, \alpha_{n}^{*}\right\}$, the characteristics of the system are $P^{\star}$. At this point, a factor can be made to vary within a certain range of the benchmark state and changes in system characteristics can be observed as the parameter changes. This analysis can be used for each factor. Therefore, the deviation degree with respect to benchmark state $P^{\star}$ of system characteristics $P$ under the changes of various factors is called parameter sensitivity analysis.

After establishing the functional relationship between system characteristic $P$ and $\mathrm{n}$ factors, it is necessary to determine the reference parameter set of $\mathrm{n}$ factors, this parameter set needs to be determined in the specific problem studied. Then, sensitivity analysis can be carried out for each influencing factor. Given a range of parameters $\alpha_{k}$ and floating within this range, the characteristics $P$ can be expressed as:

$$
P=f\left(\alpha_{1}^{*}, \cdots, \alpha_{k-1}^{*}, \alpha_{k}, \alpha_{k+1}^{*}, \cdots, \alpha_{n}^{*}\right)
$$

when each value of $\alpha_{k}$ is taken, the corresponding calculated value of system characteristic $P$ will be obtained. The analysis results are sorted out and the curves can be drawn with the results. The slope of the curve can represent the sensitivity of $P$ to parameters $\alpha_{k}$.

In fact, system characteristic $P$ is often influenced by multiple parameters, but through the above analysis, the sensitivity of system characteristic $P$ to a single factor can only be obtained. Therefore, the joint action of multiple influencing factors should be considered in the analysis of surrounding rock pressure.

According to the research results of Liu Zhuo [15] on parameter sensitivity 
analysis of surrounding rock pressure, when the surrounding rock pressure is calculated by combining the pressure arch theory, the sensitivity of internal friction Angle $\varphi$ in the sensitivity analysis is the highest, while the other high sensitivities are tunnel span $B$, rock mass weight $\gamma$ and tunnel buried depth $H$. However, the sensitivity of the cohesion $\mathcal{c}$, poisson's ratio $\mu$ and elastic modulus $E$ of surrounding rock is very low, which can be ignored in the calculation of surrounding rock pressure.

\subsection{Calculation of Surrounding Rock Pressure under the Influence of Multiple Factors}

By using parameter sensitivity analysis, it can be realized that the influence degree of rock mass density, internal friction Angle, tunnel span and buried depth is higher in the process of surrounding rock pressure calculation. However, the relationship between the above factors is not clear, and the tunnel surrounding rock pressure in actual engineering is affected by a number of factors, the calculation of surrounding rock pressure should also consider the combined effect of the above factors. Therefore, the highly sensitive tunnel depth $H$, tunnel span $B$, internal friction Angle $\varphi$ and rock mass weight $\gamma$ were introduced into the orthogonal test.

The study of complex problems often involves the analysis of the mutual influence of various factors. Orthogonal experimental design is a research method that is often used to analyze the interaction of multiple factors and simultaneously analyze multiple levels.

The method combines the orthogonality of multiple levels of these factors and selects a representative part from all the combinations. These selected experiments have the characteristic of uniform dispersion. For the analysis of multi-factor influence, orthogonal experimental design is a fast, efficient and economic research method [16].

A representative part is selected from the complete combination scheme, which is a major feature of the orthogonal experimental design, and then this part of the experiment is calculated and the results are analyzed by using statistical methods [17]. Therefore, it is necessary to select some tests with the characteristics of comparability and dispersion to form the optimal test scheme as far as possible. In 1951, Genichi Taguchi proposed the concept of orthogonal table, which can be used for orthogonal experimental design, and has the above two characteristics, which makes the orthogonal experimental design more conducive to practical application. The meaning of the symbol $L_{n}\left(q^{m}\right)$ of the orthogonal table is: $L$ is the orthogonal table, $q$ represents the number of factor levels, $m$ represents the number of columns of the orthogonal table, that is, the number of factors that can be accommodated. $n$ represents the total number of tests after combination. When calculating the surrounding rock pressure in combination with the pressure arch effect, the four influencing factors including the tunnel's buried depth $H$, tunnel span $B$, internal friction Angle $\varphi$ and rock mass weight $\gamma$ need to be taken into account. The buried depth of the tunnel is 
$50 \mathrm{~m}, 60 \mathrm{~m}, 70 \mathrm{~m}$ and $80 \mathrm{~m}$, the rock mass weight is $18 \mathrm{kN} / \mathrm{m}^{3}, 20 \mathrm{kN} / \mathrm{m}^{3}, 23$ $\mathrm{kN} / \mathrm{m}^{3}$ and $26 \mathrm{kN} / \mathrm{m}^{3}$, the internal friction angle is $25^{\circ}, 30^{\circ}, 35^{\circ}, 40^{\circ}$ and the tunnel span is $10 \mathrm{~m}, 13 \mathrm{~m}, 16 \mathrm{~m}$ and $20 \mathrm{~m}$. By looking up the orthogonal table, it was found that $L_{16}\left(4^{5}\right)$ could be used for the orthogonal design of this section. Although it has an empty column, the number of tests designed was more suitable than $L_{9}\left(3^{4}\right)$. According to the arrangement of orthogonal test table, the horizontal values of each factor are listed in Table 1. Moreover, for the insensitive factors required in the numerical simulation, the value shall be determined according to the median value of class IV surrounding rocks given in the railway tunnel design code (TB 10003-2016).

Based on Equation (3) and MIDAS/GTS NX numerical calculation, the surrounding rock pressure values of the models established by the 16 orthogonal tests in Table 1 are obtained, and the results are listed in Table 2.

Table 1. Orthogonal array table $\mathrm{L}_{16}\left(4^{5}\right)$.

\begin{tabular}{ccccc}
\hline Group & $\begin{array}{c}\text { Depth of tunnel } \\
H / \mathrm{m}\end{array}$ & $\begin{array}{c}\text { Rock mass weight } \\
\gamma /\left(\mathrm{kN} / \mathrm{m}^{3}\right)\end{array}$ & $\begin{array}{c}\text { Internal friction angle } \\
\varphi /^{\circ}\end{array}$ & $\begin{array}{c}\text { Tunnel span } \\
B / \mathrm{m}\end{array}$ \\
\hline 1 & 50 & 18 & 25 & 10 \\
2 & 50 & 20 & 30 & 13 \\
3 & 50 & 23 & 35 & 16 \\
4 & 50 & 26 & 40 & 20 \\
5 & 18 & 30 & 16 \\
6 & 60 & 20 & 25 & 20 \\
7 & 60 & 23 & 40 & 10 \\
8 & 60 & 26 & 35 & 13 \\
9 & 60 & 18 & 35 & 20 \\
10 & 70 & 20 & 40 & 16 \\
11 & 70 & 23 & 25 & 13 \\
12 & 70 & 26 & 30 & 10 \\
13 & 70 & 18 & 40 & 13 \\
14 & 80 & 20 & 35 & 10 \\
15 & 80 & 23 & 30 & 20 \\
16 & 80 & 26 & & 16 \\
\hline & 80 & & 25 & \\
\hline & & & & 25 \\
\hline
\end{tabular}

Table 2. Calculation results of surrounding-rock pressure under different parameters.

\begin{tabular}{ccccccc}
\hline Group & 1 & 2 & 3 & 4 & 5 & 6 \\
\hline Surrounding-rock pressure value/kPa & 90.28 & 123.04 & 157.14 & 203.06 & 131.98 & 159.63 \\
Group & 7 & 8 & 9 & 10 & 11 & 12 \\
Surrounding-rock pressure value/kPa & 104.09 & 151.27 & 138.13 & 144.62 & 132.66 & 138.78 \\
Group & 13 & 14 & 15 & 16 & & \\
Surrounding-rock pressure value/kPa & 98.96 & 103.08 & 184.63 & 192.39 & &
\end{tabular}




\subsection{Multiple Linear Regression Analysis of Surrounding Rock Pressure}

Based on the calculated results of surrounding rock pressure, regression analysis method can be used for mathematical statistical analysis. Regression analysis is to establish a statistical analysis model based on the results of orthogonal experiment, which can be used to study the relationship between variables and establish an approximate relation to control and predict variables. Multiple linear regression analysis can be used to analyze the linear relationship of multiple variables. According to the numerical results of the orthogonal experiment, this method is used to make mathematical statistics analysis, and the practical calculation formula of surrounding rock pressure is obtained.

Four influencing factors involved in the orthogonal experiment were selected: tunnel depth $H$, tunnel span $B$, internal friction Angle, and rock mass weight, respectively, to establish the multiple linear regression equation:

$$
p=\beta_{0}+\beta_{1} H+\beta_{2} B+\beta_{3} \varphi+\beta_{4} \gamma
$$

$\beta_{1}, \beta_{2}, \beta_{3}$ and $\beta_{4}$ are partial regression coefficients, $\beta_{0}$ is the constant term set for linear regression.

The multiple linear regression function of MATLAB analysis software was used to solve the coefficients of the above regression model. The linear regression equation can be obtained by substituting into the data model:

$$
p=-83.6625+0.1758 H+6.4003 B-0.5789 \varphi+6.6057 \gamma
$$

$R$ test, $F$ test and $t$ test were used to calculate the $R$ value of 0.934 by MATLAB, which was close to 1 , indicating that the correlation coefficient was significant. $F$ value was calculated to be 58.9724 , and given a significant level of $\alpha=0.05, \quad F_{\alpha}=2.93$ was obtained by looking up the $F$ distribution table. $F>F_{\alpha}$ means that the linear assumption is valid. Calculate the $T$ value of the coefficient before each independent variable, namely $T(1)=5.493, T(2)=10.871$, $T(3)=-3.152, T(4)=2.846$. Given a significance level of $\alpha=0.05$, look up the $t$ distribution table to get a critical value $T_{\alpha}=2.1448$.

It can be known that $\left|T_{i}\right| \geq T_{a}$, then the regression coefficient $\beta_{i}(i=1,2,3,4)$ has significant significance, and the regression equation should be retained for each variable.

\section{Application Examples}

Chongqing metro underground station was selected for analysis and verification. The tunnel span of the station is $23.30 \mathrm{~m}$, the buried depth is $35 \mathrm{~m}$, and the surrounding rock level is IV. The station is constructed by double-wall guide pit method, and the core rock is curved with a minimum thickness of $6.90 \mathrm{~m}$ and a width of $8.20 \mathrm{~m}$ on both sides. The construction sequence is shown in Figure 1 . When the surrounding rock pressure affected by the construction process is taken into account, the tunnel section is simplified to some extent. The simplified model is shown in Figure 2. 


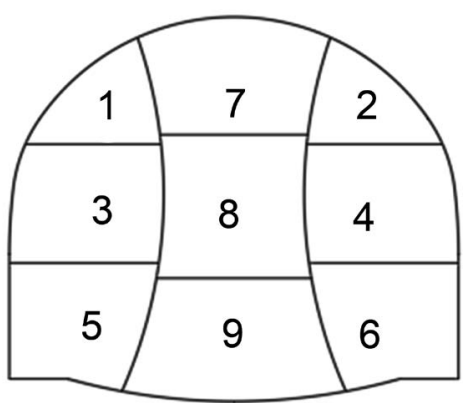

Figure 1. Excavation step of the staion.

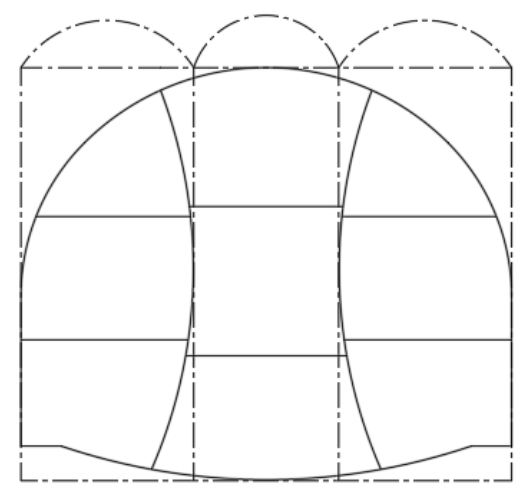

Figure 2. The model of simplified calculation.

By substituting the rock mass weight $\gamma$, tunnel depth $H$, tunnel span $B$ and internal friction Angle $\varphi$ of the example station into formula (9), the basic surrounding rock pressure values of the three guide tunnels under the pressure arch theory can be obtained. $p_{1}=p_{2}=128.27 \mathrm{kPa}$, the surrounding rock pressure value of the middle guide tunnel is $p_{3}=119.95 \mathrm{kPa}$.

\section{Conclusion}

The surrounding rock pressure is mainly composed of the loosening pressure of the lower rock mass of the pressure arch and the deformation pressure of the rock mass inside the pressure arch, which acts on the support structure. Based on the theory of pressure arch, the calculating formula $p=p_{1}+p_{2}=\gamma \cdot H_{1}+k \cdot s$ of surrounding rock pressure is deduced. By using the method of sensitivity analysis, through orthogonal experiments and regression analysis, the pressure calculation formula of subway station tunnel with a large section is established: $p=-83.6625+0.1758 H+6.4003 B-0.5789 \varphi+6.6057 \gamma$. It is also applied to the underground excavation of subway station using double-side guide pit method, and the correct surrounding rock pressure value is obtained. It is of guiding significance for the theoretical analysis and engineering practice of surrounding rock pressure in large section tunnel.

\section{Acknowledgements}

This research is funded by the China Construction Tunnel Construction Co., 
Ltd. Technology Plan Project.

\section{Conflicts of Interest}

The authors declare no conflicts of interest regarding the publication of this paper.

\section{References}

[1] Gu, Z.Q., Peng, S.Z. and Li, Z.K. (1994) Underground Chamber Engineering. Tsinghua University Press, Beijing.

[2] Shen, M.R. (1991) Rock Mechanics. Tongji University Press, Shanghai.

[3] Sun, J. (2014) Research and Application of Key Technology in Tunnel Structure Design. People's Communications Press, Beijing.

[4] Huang, Z.P., Broch, E. and Lu, M. (2002) Cavern Roof Stability-Mechanism of Arching and Stabilization by Rock Bolting. Tunnelling and Underground Space Technology, 17, 249-261. https://doi.org/10.1016/S0886-7798(02)00010-X

[5] Liang, X.D., Liu, G. and Zhao, J. (2005) Definition and Analysis of Arching Action in Underground Rock Engineering. Journal of Hohai University (Natural Sciences), No. 3, 314-317.

[6] Yu, B. and Wang, H.J. (2008) Study on the Theory of Pressure Arch and the Classification Method of Tunnel Buried Depth. China Railway Press, Beijing.

[7] Wang, Y.F., Li, C.H., Zheng, X.J. and Xia, S.W. (2009) Research on the Evolution Mechanism of Pressure Arch of Underground Cavern Excavated in Different Ways. Mining $R \& D$, No. 3, 12-14.

[8] Li, K. (2010) Pressure Arch Theory Study of Horizontal Bedded Tunnel Surrounding Rock. Doctor Degree Dissertation, Southwest Jiaotong University, Chengdu.

[9] Lee, C.J., Wu, B.R. and Chen, H.T. (2006) Tunnel Stability and Arching Effects during Tunneling in Soft Clayey Soil. Tunnelling and Underground Space Technology, 21, 119-132. https://doi.org/10.1016/j.tust.2005.06.003

[10] Wu, J.H., Ohnishi, Y. and Nishiyama, S. (2004) Simulation of the Mechanical Behavior of Inclined Jointed Rock Masses during Tunnel Construction Using Discontinuous Deformation Analysis (DDA). International Journal of Rock Mechanics \& Mining Sciences, 41, 731-743. https://doi.org/10.1016/j.ijrmms.2004.01.010

[11] Shen, B. and Barton, N. (1997) The Disturbed Zone around Tunnels in Jointed Rock Masses. International Journal of Rock Mechanics \& Mining Sciences, 34, 117-125. https://doi.org/10.1016/S1365-1609(97)80037-8

[12] Callari, C. (2004) Coupled Numerical Analysis of Strain Localization Induced by Shallow Tunnels in Saturated Soils. Computers and Geotechnics, 31, 193-207. https://doi.org/10.1016/j.compgeo.2004.01.004

[13] Bhasin, R.K., Barton, N. and Grimstad, E. (1999) Comparison of Predicted and Measured Performance of a Large Cavern in the Himalayas. International Journal of Rock Mechanics and Mining Sciences \& Geomechanics Abstracts, 33, 607-626. https://doi.org/10.1016/0148-9062(95)00082-8

[14] Martino, J.B. and Chandler, N.A. (2004) Excavation-Induced Damage Studies at the Underground Research Laboratory. International Journal of Rock Mechanics \& Mining Sciences, 41, 1413-1426. https://doi.org/10.1016/j.ijrmms.2004.09.010

[15] Liu, Z. (2014) The Mechanism Research of Arch Tunnel Surrounding Rock Pressure. Tiedao University, Shijiazhuang. 
[16] Jiang, T.C. (1985) Orthogonal Experimental Design. Shandong Science and Technology Press, Jinan.

[17] He, X.L. (2013) Research on Self-Stability of Surrounding Rock Based on Strength Reduction Method. Hunan University, Changsha. 